\title{
Auditory system multi-unit activity and behavior in the rat ${ }^{1}$
}

THOMAS J. IMIG ${ }^{2}$ and NORMAN $M$. WEINBERGER, Department of Psychobiology, University of California, Irvine, Calif. 92664

Multiple-unit activity (MUA) in the auditory system was measured during the behavior of water-deprived rats. MUA varied significantly and increased monotonically across "resting," "sniffing," "exploring," "drinking," and "grooming." The results indicate that $M U A$ level associated with ambient behavior must be taken into account in studies seeking $M U A$ correlates of learning or of other behavioral processes.

In recent years, the number of investigations seeking relationships between neuronal unit activity and organismic behavioral processes (e.g., learning, perception) or states (e.g., sleep) has increased greatly. In particular, recording the action of multiple units with a single electrode is becoming increasingly popular. Multiple-unit activity (MUA) has been reported to vary with state; it is greatest during paradoxical sleep, lower during waking, and lowest during slow-wave sleep in the thalamus and reticular formation (Winters et al, 1967; Podvall \& Goodman, 1967). Within the waking state, MUA in the reticular formation decreases during behavioral habituation (Weinberger et al, 1969). MUA during the waking state is of particular interest because studies of learning, selective attention, habituation, and other behavioral processes ordinarily take place within this background state. However, there are no systematic studies of the relationship between MUA and waking behaviors. The present study was undertaken as a prelude to an investigation of MUA in the auditory system of rats during behavioral habituation. ${ }^{3}$

\section{METHODS}

The Ss were four adult male albino rats (250-350 g). Two to 4 weeks prior to the first experimental session, each received electrodes chronically implanted in the lateral lemiscus or inferior colliculus (later verified histologically). Electrodes were bipolar, consisting of insect pins with tips of 30-50 microns, insulated except for $0.5 \mathrm{~mm}$ of shank from the tip and offset $1 \mathrm{~mm}$ vertically. Standard surgical implantation and postoperative techniques were used throughout.

The apparatus consisted of a Plexiglas box, $30 \times 30 \times 30 \mathrm{~cm}$, with a grid floor. An inverted graduated cylinder was mounted on the outside of one wall, with the drinking spout protruding $1.5 \mathrm{~cm}$ into the box, 4 in. above the floor. A clear Plexiglas partition could be inserted from above to divide the box and prevent access to the drinking tube. The testing box was housed in a double acoustic chamber (IAC No. 1202) with a one-way observation window.

The animals were deprived of water for $48 \mathrm{~h}$ prior to an experimental session. An animal was placed in the testing box with the partition preventing access to the tube and connected to the recording cable. At the end of $2 \mathrm{~min}$, the partition was removed, and all animals located the tube and began drinking. At the end of $2 \mathrm{~min}$, the partition was replaced, and thereafter, 2 -min periods of access alternated with 2 -min periods of nonaccess until the rat did not lick the water tube during two consecutive periods. Animals were deprived and tested several times over periods ranging from 1 to 4 weeks between successive sessions. A total of 21 sessions were run with the four rats; however, data from only 18 are reported because the analysis criterion (see below) was not satisfied on three sessions of Rat 1. (Ss and number of sessions were: No. 1, 1; No. 2, 6 ; No. 3,5 ; No. 4, 6.)

The animal was observed continuously, its behavior being assigned to one of the following categories: "resting," "sniffing," "exploring," "drinking," and "grooming." Categorization was accomplished without knowledge of the level of MUA, which was recorded simultaneously. Behavioral category together with a signal coincident with its duration were recorded on a polygraph. Later analysis was restricted to behaviors that were of at least $2 \mathrm{sec}$ duration without a break, and at least 10 such epochs of each behavior were used to compute the mean MUA activity. MUA was quantified by filtering neuroelectric activity to pass $800-6 \mathrm{~K} \mathrm{~Hz}$ through a Schmitt trigger, set at 10 microvolts. Standard output pulses from the trigger were "integrated" with an RC circuit, and the resultant voltage was recorded on the polygraph (T.C., $1 \mathrm{sec}$ ). MUA level during each behavior was taken as the mean voltage during a 2 -sec epoch, determined by hand measurement every $200 \mathrm{msec}$.

\section{RESULTS}

Inspection of "raw" multi-unit activity indicates that it is different during the various behaviors (Fig. 1). The most obvious finding is that MUA is least during "resting" and greatest during "grooming." The relationship between MUA and behavior was quantified by ranking the behaviors within each session according to the mean amount of MUA present. 4 Friedman two-way analyses of variance showed significant effects of behavior for the three rats with multiple sessions and also for the pooled data $\left(\chi^{2} \mathrm{r}=37.1, \mathrm{df}=4, \mathrm{p}<.001\right)$. If the behaviors are listed according to an a posteriori presumption about the amount of gross movement associated with each behavior, then MUA in the auditory system was found to increase as a function of increasing amounts of movement (Fig. 2). Sign tests showed that all behavioral categories differed significantly from each other $(\mathrm{N}=18, \mathrm{p}<.015)$ except "drinking" and "grooming."

\section{DISCUSSION}

The present findings demonstrate that MUA in the auditory system changes significantly during the differing behaviors that were investigated here. There appears to be a monotonic increasing relationship between the amount of MUA and the amount of gross movement associated with each behavior. Validation of this point, however, will require a study that uses an independent measure of "gross movement." MUA might also reflect general arousal level. Whatever the

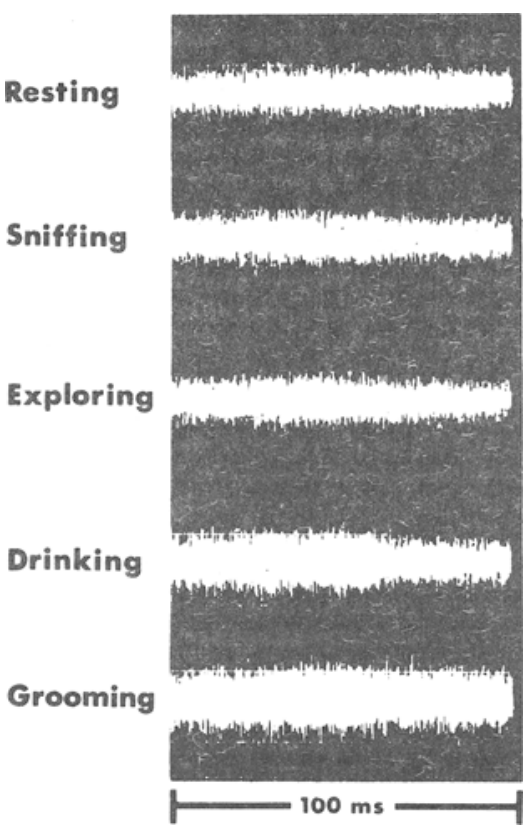

Fig. 1. Multiple-unit activity recorded from the ventral inferior colliculus near the entrance of the lateral lemniscus from one rat. 


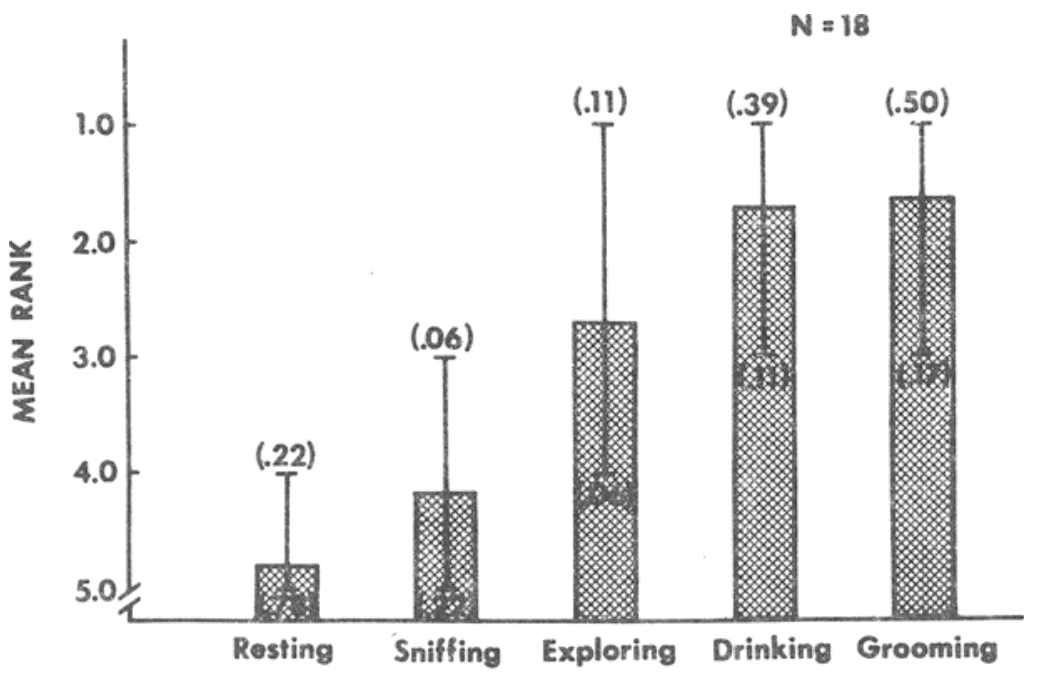

\section{BEHAVIOR}

Fig. 2. Mean ranks of "integrated" multiple-unit activity for all scssions (vertical bars); the greatest amount of MUA received a rank of "1," etc. The vertical lines denote the range of ranks found for each behavior, and the numbers in parentheses indicate the proportion of times that these extreme. ranks were reached.

underlying cause of the relationship seen here, it is clear that MUA level in the auditory system is not independent of ongoing behavior in the waking state. An important implication of this finding is that investigations of behavioral processes (e.g., learning) that employ MUA may confound a performance correlate with a presumed "process" correlate (e.g., "learning") unless the effect of ambient behavior itself is controlled or taken into account.

\section{REFERENCES}

WEINBERGER, N. M., GOODMAN, D. A., \& KITZES, L. M. Is behavioral habituation a function of peripheral auditory system blockade? Communications in Behavioral Biology, 1969, 3, 111-116.

PODVALL, E. M., \& GOODMAN, S. J. Averaged neural electrical activity and arousal. Science, 1967, 155, 223-225.

WINTERS, W. D. MORI, $K$, SPOONER, C. E., \& KADO, R. T. Correlation of reticular and cochlear multiple unit activity with auditory evoked responses during wakefulness and sleep: 1. Electroencephalography \& Clinical Neurophysiology, 1967, 23, 539-545. NOTES

1. Supported by Research Grant MH 11250 from the National Institute of Mental Health to N. M. Weinberger.

2. Trainee supported by Grant No. 11095 from the National Institute of Mental Health.

3. Weinberger, N. M., \& Imig, T. J. Auditory system evoked activity during habituation of orientation in the rat. In preparation.

4. Ranking, rather than conventional analysis of variance, was used because the voltage output of the integrator while being monotonically related to spike rate was not linearly related across the entire range of unit activity.

\section{Roughness discrimination with sandpaper surfaces: An olfactory confounding}

\section{STANLEY FINGER, Washington University, St. Louis, Mo. 63130, GABRIEL P. FROMMER, Indiana University, Bloomington, Ind. 47401, and AMIRAM CARMON and RIFKA INBAL, Hebrew University Medical Center, Jerusalem, Israel}

Enucleated rats were tested for acquisition of a rough-smooth discrimination in which the front and back surfaces of a piece of sandpaper served as discriminanda. Either lesions or
1959; Finger \& Frommer, 1968a; Lovett, 1935; Semmes \& Mishkin, 1965; Smith, 1939; Zubek, 1952). Sometimes, the abrasive discriminandum was paired with the surface of some other material (e.g., cardboard). In other experiments, the coarse side of the sandpaper was paired with the smooth, back surface of the same material in an attempt to eliminate the differential olfactory cues that would be present if two different materials served as discriminanda. In the present study, it is shown that even this procedure is inadequate to eliminate such olfactory confounding, at least in rats.

\section{EXPERIMENT 1}

Methods

Six naive male rats, weighing $225.325 \mathrm{~g}$, served as Ss in the first experiment. All animals were enucleated under pentobarbital sodium (Nembutal) and were adapted to a 23-h food-deprivation schedule 2 weeks prior to the start of testing.

Apparatus and testing procedure were the same as those described previously (Finger \& Frommer, 1968a). Briefly, a T-maze was used for discriminative testing. Front and back sides of a relatively coarse paper (Grade 360A aluminum oxide paper, Carborundum Co.), running on the floor of the maze from the choice point to the foodcups, served as rough-smooth discriminanda. Three Ss were assigned to be rewarded for choosing the rough surface, while the remaining rats received food for choosing the smooth surface. During testing (five trials/day), the tactile surfaces were shifted in the maze according to a semirandom procedure, so that the same stimulus did not appear more than three times in a row on a given side. Although both wings of the maze contained food, a screen prevented animals from eating after an incorrect choice.

After 150 trials of the initial task, a series of changes in the discriminanda was instituted to assess the roles of tactile and olfactory cues in guiding behavior. For blocks of 25 trials, one, or the other, or both possible discriminative cues were reduced. In the first manipulation, aluminum window screening was placed over both pieces of sandpaper so that tactile cues from the abrasive surfaces, but not olfactory cues, were diminished. Next, a deodorizer spray (Wizard) was applied to the sandpaper, and the screening was put over it, so that both tactile and potential olfactory cues were diminished. In the following block, the mesh was removed, and only spray was applied to the sandpaper. The original conditions were then reinstated to test whether or not disruptions in performance could be attributed to stimulus change. 